\title{
1 Prey identity affects fitness of a generalist consumer in a brown food web
}

2 Authors: Lily Khadempour ${ }^{1,2}$, Leslie Rivas Quijano ${ }^{1}$, Casey terHorst ${ }^{1}$

3

4 Affiliations:

$5 \quad{ }^{1}$ Department of Biology, California State University, Northridge, Northridge, CA, 91330, USA

$6{ }^{2}$ Present address: Department of Earth and Environmental Sciences, Rutgers University, Newark,

$7 \quad$ Newark, NJ, 07102, USA

8 


\section{Abstract}

11 The use of ever-advancing sequencing technologies has revealed incredible biodiversity at the

12 microbial scale, and yet we know little about the ecological interactions in these communities.

13 For example, in the phytotelmic community found in the purple pitcher plant, Sarrecenia

14 purpurea, ecologists typically consider the bacteria as a functionally homogenous group. In this

15 food web, bacteria decompose detritus and are consumed by protozoa that are considered

16 generalist consumers. Here we tested whether a generalist consumer benefits from all bacteria

17 equally. We isolated and identified 22 strains of bacteria, belonging to six genera, from $S$.

18 purpurea plants. We grew the protozoa, Tetrahymena sp. with single isolates and strain mixtures

19 of bacteria and measured Tetrahymena fitness. We found that different bacterial strains had

20 different effects on protozoan fitness, both in isolation and in mixture. Our results demonstrate

21 that not accounting for composition of prey communities may affect the predicted outcome of

22 predator-prey interactions.

24 Keywords: Sarrecenia purpurea, phytotelmic, predator-prey dynamics, bottom-up effects

\section{Introduction}

Predator-prey or consumer-resource dynamics are among the best studied ecological

28 interactions. Theory suggests that coexistence of predator and prey depends on several

29 parameters that incorporate traits of both predator and prey, such as attack rate, handling time,

30 and conversion efficiency (Holling 1959). Considerable theory has explored how variability of

31 predator and prey traits affects population dynamics, demography, and species coexistence (e.g.

32 Abrams and Rowe 1996; Kendall et al. 1999; Peckarsky et al. 2008; Fleischer et al. 2018). Many 
33 empirical examples demonstrate how different prey traits affect handling time (e.g. Werner 1974;

34 Faria et al. 2004) and attack rates of predators (Boates and Goss-Custard 1992; Elliott 2003)

35 ultimately having consequential effects on predator fitness. It would come as no surprise to many

36 ecologists that the identity of an animal's prey affects performance and fitness, yet in the

37 microbial world, we have far less understanding of the specificity of trophic interactions.

Most examples of trophic interactions emerge from green food webs, where

39 photosynthetic plants or algae form the base of the food web. However, brown food webs, in

40 which detritivores, such as bacteria and fungi, form the base of the food web, are also common in

41 nature. In green food webs, ecologists are keenly aware of how traits of the plant community

42 affect the transfer of energy through a food web (Stap et al. 2007; Mooney et al. 2010).

43 However, in brown food webs, the species composition of the base of the food web has

44 historically been treated as a black box where "bacteria" or "fungi" are treated as a single

45 taxonomic unit (eg. Miller and terHorst 2012). Despite rapid advances in identifying bacterial

46 taxa in natural communities over the past decade, we still have far less knowledge of the

47 ecological role of specific taxa and how they interact with other species. The effect of different

48 bacterial species on consumer growth rates has remained largely untested (but see Mohapatra

49 and Fukami 2005; Darby and Herman 2014), even though variation in bacterial species traits are

50 likely to alter consumer attack rates, handling times, and conversion efficiencies.

The phytotelmic (organisms that inhabit small pools of water within or upon plants)

52 community found in the leaves of the purple pitcher plant (Sarrecenia purpurea) has been used

53 as a model system for studying broad questions about ecology and evolution (Miller et al. 1994;

54 Cochran-Stafira and Ende 1998; Ellison and Gotelli 2002; Kneitel and Miller 2002; Miller et al.

55 2014). The pitcher-shaped leaves attract insects and serve as pitfall traps in which insects drown, 
56 decompose, and provide nutrients to the plant. The insects serve as the source of energy and

57 nutrients at the bottom of a brown food web. Bacteria decompose the dead insects and are

58 consumed by a suite of protozoa and rotifer species, which are consumed by mosquito larvae.

59 Numerous top-down studies have demonstrated that the evolutionary and ecological dynamics of

60 protozoan consumers affect bacterial community composition (Cochran-Stafira and Ende 1998;

61 Peterson et al. 2008; Paisie et al. 2014; Holdridge et al. 2016; Canter et al. 2018). These top-

62 down effects should not be surprising since protozoans have demonstrated selective feeding

63 behavior in other systems (Strom and Loukos 1998; Gaines et al. 2019). Selective feeding clearly

64 has an effect on prey community dynamics, and it also suggests that different prey could have

65 different effects on consumer fitness. However, there is considerably less work on the bottom-up

66 effects of different bacterial species on protozoan ecological dynamics. Although some studies

67 have examined the effects of total bacterial abundance on higher trophic levels (e.g., Kneitel and

68 Miller 2002; Hoekman 2007), most studies indirectly manipulate the bacterial community as a

69 whole by altering resource availability, rather than particular taxa of bacteria with different traits.

70 An underlying assumption with these studies is that the protozoa are generalist consumers of

71 bacteria and that, regardless of their identity, a higher abundance of bacteria promotes protozoan

72 growth.

73 Here we examine this assumption and ask whether different strains of bacteria

74 differentially affect consumer fitness. We collected fluid from $S$. purpurea pitcher plants found

75 in the field, from which we isolated single strains of bacteria. We quantified the effects of single

76 strains and multi-strain communities of bacteria on the fitness of a common ciliate (Tetrahymena

77 sp.), which is found in S. purpurea phytotelmic communities, and is commonly used in lab

78 microcosm experiments in this system. 


\section{Methods}

\section{$80 \quad$ Isolation of bacteria}

81 We collected water contained within pitcher plant leaves haphazardly from leaves of

82 various ages in one field in the Apalachicola National Forest in northern Florida (USA). Large

83 insect parts were filtered from the fluid and $2 \% \mathrm{v} / \mathrm{v}$ DMSO was added before freezing at $-20^{\circ} \mathrm{C}$

84 in $50 \mathrm{~mL}$ conical tubes. Samples were shipped to California State University, Northridge, where

85 we thawed the tubes and diluted the liquid with sterilized water at 10x, 100x, and 1000x

86 dilutions. We spread $50 \mu \mathrm{L}$ of liquid from each dilution onto LB solid media plates (Cold Spring

87 Harbour Protocols 2016) using a plate spreader. We monitored these plates daily to check for

88 bacterial colony growth and then identified different morphotypes of bacteria, which were picked

89 and streaked in order to isolate individual strains. Once we were confident the bacteria were in

90 isolation, both through visual confirmation and sequencing (see below), we maintained them in 5

$91 \mathrm{~mL}$ of liquid LB media (Cold Spring Harbour Protocols 2016) and transferred them to fresh

92 tubes every two weeks.

\section{Identification of bacteria}

94 We extracted DNA from bacterial strains using the Qiagen Blood and Tissue Kit, with

95 the specifications for bacterial cultures. We used $16 \mathrm{~S}$ rRNA gene primers $27 \mathrm{~F}$ and $1392 \mathrm{R}$ in a

96 PCR with the following conditions: $94^{\circ} \mathrm{C}$ for $5 \mathrm{~min}, 30$ cycles of $94^{\circ} \mathrm{C}$ for $20 \mathrm{~s}, 55^{\circ} \mathrm{C}$ for $20 \mathrm{~s}$,

97 and $72^{\circ} \mathrm{C}$ for $70 \mathrm{~s}$, with a final elongation of $72^{\circ} \mathrm{C}$ for $10 \mathrm{~min}$. We sequenced the amplicons with

98 Sanger sequencing through Laragen (Culver City, CA) with both forward and reverse primers,

99 then trimmed, identified, and analyzed sequences using 4Peaks and CLC Sequence Viewer 7.

100 We used NCBI BLAST to identify the strains to the genus level using their 16S sequences.

101 Ultimately, we isolated 22 individual unique strains of bacteria (Table S1). 


\section{Isolation of protozoa}

103

For several years, we have maintained eight strains of the ciliated protozoa Tetrahymena

$104 \mathrm{sp}$. in lab cultures. These strains were originally collected from different pitcher plants in the

105 Apalachicola National Forest and have been maintained independently in the lab. For this experiment, we isolated each of these strains from their associated bacterial community. We

107 created YPD sterile media (YPD media 2010) supplemented with four filter-sterilized antibiotics

108 (final concentration in parentheses): kanamycin $(50 \mathrm{mg} / \mathrm{mL})$, tetracycline $(10 \mathrm{mg} / \mathrm{mL})$, penicillin

109 (1200 mg/mL), and streptomycin $(120 \mathrm{mg} / \mathrm{mL})$. We added $100 \mu \mathrm{L}$ of each Tetrahymena strain to

110 separate replicate test tubes with media and allowed them to grow for three days at room

111 temperature. We then looked for bacteria in a small volume of the protist culture at 1000x

112 magnification. We only used replicate tubes in which little or no bacteria were visible. This

113 technique was unlikely to have removed all bacteria, but the concentration of bacteria in these

114 cultures was many orders of magnitude lower than the bacteria that we added in the experimental 115 tubes below. We then added each protist strain to the experimental tubes, as described below.

\section{Effect of individual bacterial strains}

117 We established microcosms by adding $10 \mathrm{mg}$ of crushed Tetracolor Fish Flakes to $10 \mathrm{~mL}$

118 of water in a test tube before autoclaving for $45 \mathrm{~min}$ at $120{ }^{\circ} \mathrm{C}$. We added $30 \mu \mathrm{L}$ of liquid culture

119 of an individual bacterial strain to each tube and allowed these bacteria to establish and grow for

120 one day at ambient room temperature. We did not control for bacterial abundance as we

121 considered bacterial growth rate on the media as one of several bacterial traits that could

122 potentially differ among strains. We then added an individual Tetrahymena strain to different

123 tubes, so that each Tetrahymena strain was grown with each bacterial strain; each Tetrahymena

124 strain served as a replicate $(n=8)$ in testing the effects of the bacterial strains. The initial density 
125 of protozoa in each tube was 100 individuals per $\mathrm{mL}$. We allowed the bacteria and protozoa to

126 grow together for four days at ambient room temperature, and then counted the density of

127 protozoa using Palmer cells (Wildlife Supply Company, Yulee, Florida, USA).

128

Effect of multiple bacterial strains

To determine whether different bacterial strains would have different effects when

131 combined with other bacterial strains in a community context, we also created synthetic

132 communities of bacteria. We created five different combinations of six bacterial strains. From

133 the first experiment, we chose (1) the six best strains, in terms of their effect on protozoan

134 fitness, (2) the six worst strains, (3) the three best and three worst strains, (4) the two best, two

135 worst, and two intermediate strains, and (5) a combination of the six worst strains that had

136 different morphotypes (Table S2). We grew each of these five bacterial combinations with each

137 of the eight unique Tetrahymena strains. We mixed equal volumes of the six strains in each

138 combination and then aliquoted $30 \mu \mathrm{L}$ of this mixture into each replicate experimental tube. We

139 allowed these bacterial cultures to grow for one day before adding protozoa and quantifying their

140 growth in the same way as in the previous experiment.

141 For both experiments, we used ANOVA to examine the effects of bacterial strain or

142 mixture on the final abundance of protozoa. Tetrahymena strains were considered as independent

143 replicates. We followed significant treatment effects with Tukey's HSD to examine pairwise

144 differences among treatment levels, using the 'agricolae' package (Mendiburu and Yaseen 2020)

145 in R 3.6.3 (R Core Team 2013).

\section{Results and Discussion}


In total, we isolated 22 bacterial strains with different morphotypes, belonging to six genera: Serratia, Chromobacterium, Chryseobacterium, Burkholderia, Acinetobacter, and

Bacillus. Individual strains of bacteria had different effects on protozoan fitness $\left(\mathrm{F}_{(21,153)}=14.2\right.$,

$150 \mathrm{P}<0.001$ ); Figure 1). Some bacterial taxa resulted in highly abundant protozoan populations,

151 although other taxa either could not support protozoan growth or actively inhibited it, and many

152 other taxa had varying degrees of intermediate effect.

154 decidedly not a generalist with regard to the benefits it receives from consuming prey. Instead,

155 bacterial taxa reside on a broad spectrum in regard to their effects on protozoan fitness. Although

156 many studies in this system assume that protozoan fitness is primarily determined by the

157 abundance of bacterial prey, our results suggest that fitness may be largely determined by the 158 composition of the bacterial community.

Two Chromobacterium strains (1F and 1I) had especially negative effects on protozoan

160 fitness, with nearly no protozoa observed in these cultures. This is consistent with previous

161 studies that have shown that Chromobacterium spp. inhibit protozoan growth through the

162 production of the pigment violacein (Singh 1945; Pickup et al. 2007). For this reason,

163 Chromobacterium has been targeted as a potential source of compounds for treating fungal and

164 viral infections, as well as cancer cell growth (Cheng et al. 2007; Sasidharan et al. 2015).

165 However, other strains of Chromobacterium (4B, 3G, and 2C) produced some of the highest

166 protozoan abundances. This emphasizes that the ecological function of bacteria cannot be

167 generalized, even among closely-related taxa; single strains of bacteria may not well represent

168 the effects of other species in the same genus. Other genera, such as Burkholderia and Serratia,

169 had consistently positive effects on protozoan fitness, although more isolation and testing of 
170 other taxa within these genera are necessary to determine if this is generally true. The effects on

171 bacteria traits on protozoa growth are not limited to only toxicity, as there were a range of

172 intermediate effects of different bacteria taxa. Other factors such as, but not limited to, growth

173 rate, motility, and nutritive quality might also affect protozoan fitness, and a more detailed

174 analysis of bacterial traits and their effects on protozoans would provide for interesting future

175 work (Goyal et al. 2021).

176 Our pairwise interaction experiment demonstrated that bacterial effects on protozoan

177 fitness depend on bacterial strain identity. However, in natural communities, protozoa are

178 unlikely to interact with one bacterial species in isolation. In our second experiment, we found

179 that different community compositions had different effects on protozoan abundance $\left(\mathrm{F}_{(4,34)}=\right.$

180 29.5, P < 0.001; Figure 2). The negative effects of the "bad" bacterial strains (those that had

181 strong detrimental effects on protozoan fitness) persisted in a community context, where the

182 effects of bad strains outweighed the positive effects of relatively "good" strains (those that have

183 relatively beneficial effects on protozoan fitness). Any combination that included the two bad

184 bacteria from the first experiment (1F and 1I) resulted in low protozoan fitness (Figure 2). This

185 pattern is consistent with bad strains having produced toxins with effects that were not dampened

186 by the presence of other species, rather than the bad strains not supplying sufficient nutrition for

187 the protozoa. It is important to note that our synthetic communities only consisted of six bacterial 188 strains, which is considerably less diverse than a natural community found in a pitcher plant leaf

189 (Koopman et al. 2010; Koopman and Carstens 2011; Gray et al. 2012), reported to contain

190 approximately 400 bacterial species (Paisie et al. 2014). It is unclear whether the negative effects

191 of the bad bacteria would be dampened in a more diverse bacterial community in a pitcher plant 
192 leaf, which has many more microhabitats than a test tube, and where the bad bacterial species

193 would face greater and more diffuse competition for resources with other bacteria.

195 break down insects and release nutrients, while protozoa are thought to be parasitic because they consume bacteria (Mouquet et al. 2008). Our results suggest that these categorizations may be

197 more complex since protozoa do not necessarily reduce the abundance of all bacterial taxa

198 evenly. This result also supports previous work in this system and others that demonstrates that

199 protozoa have different prey selection patterns (Strom and Loukos 1998; Gaines et al. 2019).

200 Furthermore, presumably some bacterial taxa break down prey faster than others, although this

201 has not been tested in this system, to our knowledge, nor do we know whether there is a trade-off

202 between this function and susceptibility to predation. We also do not yet know whether the same

203 taxa-specific effects of bacteria affect other protozoan consumers in the same way. Future work

204 could expand on these results by doing similar manipulations inside pitcher plant leaves and

205 examining insect degradation rates or nutrient uptake by the plant. Such information would help

206 us to understand how dynamics among species within the phytotelmic community ultimately

207 affect the plant in which they live.

When Tetrahymena protozoa feed, they appear to ingest any bacteria in their immediate

209 surroundings, although they often cluster around structures in the water (e.g., insect parts or

210 pieces of detritus; personal observation). The extent to which bacteria growing on structures in

211 the water differ from those in the water column, or whether good and bad bacteria differ between

212 these environments is unknown and would be an interesting avenue for future work in this

213 system. It has been established that protozoa have top-down effects on bacterial community

214 composition (Cochran-Stafira and Ende 1998; Peterson et al. 2008; Paisie et al. 2014; Holdridge 
et al. 2016; Canter et al. 2018), and this, combined with the knowledge that different strains

216 affect protozoan fitness, would suggest that the protozoa may engage in a more active form of

217 prey selection than previously thought. There are, however, alternative explanations for the top-

218 down effects and prey selection patterns of the protozoa on bacterial community composition.

219 For example, some bacterial taxa may have faster growth rates, and so can recover more quickly

220 from grazing, or some bacteria may congregate in microniches where protozoa are more

221 abundant, making them more likely to be eaten.

Overall, our results demonstrate that bacterial prey identity affects consumer fitness and

223 that it is vital that future work in these pitcher plant communities account for bottom-up effects

224 of different bacterial taxa on protozoan growth and fitness, both by taking this into account in

225 models, but also by considering this in empirical studies. For example, Miller et al. (2012) found

226 only a weak relationship between bacterial abundance and protozoan abundance across a

227 successional sequence in pitcher plant leaves. However, our work suggests that examining

228 relationships between particular types of bacteria and their effects on the abundance of different

229 protozoa species, and vice versa, could reveal previously unrecognized ecological patterns.

230 Future work could consider how to manipulate or quantify the bacterial community in such a

231 way to tease apart these dynamics. These results open up new avenues of research in pitcher

232 plant microcosms that allow for the study of bottom-up effects by maintaining lab protozoa

233 aseptically, or with known bacterial taxa, allowing for manipulation of the bacterial community

234 during lab experiments. Alternatively, researchers who either create their own synthetic

235 communities or identify bacterial community members through shotgun sequencing can be

236 aware of which bacterial taxa are present and can account for those effects appropriately, rather

237 than treating the bacterial community as a single unit. 
Like other brown food webs, pitcher plant microbial communities are, in practice, often

239 treated as food chains, where the bacteria at the base of the food web are assumed to be

240 functionally redundant. This study demonstrates that this assumption is not valid, and that when

241 bottom-up effects are being examined in this system, it is important to consider the identity of

242 the bacterial community members and their effects on the predators that feed on them. We

243 believe that the results of this study can be further extrapolated to other brown food webs,

244 suggesting that we must move away from treating the foundation of these webs as a black box.

\section{Acknowledgements}

247 The authors would like to thank T. Miller and C. Cuellar-Gempeler for their help in collecting

248 bacteria from the field and members of the CSUN EcoEvo Lab for their feedback on an earlier

249 version of the manuscript. This project was funded by grants from the National Science

250 Foundation to CPT (OCE-1559105 and DEB-1754449). Author contribution statement

251 Conceived of the idea: LK and CPT. Conducted the experiment: LK and LRQ. Wrote the

252 manuscript: LK, LRQ, and CPT. 


\section{References}

255 Abrams, P. A., and L. Rowe. 1996. The effects of predation on the age and size of maturity of

256 prey. Evolution 50:1052-1061.

257 Boates, J. S., and J. D. Goss-Custard. 1992. Foraging behaviour of oystercatchers Haematopus

258 ostralegus specializing on different species of prey. Canadian Journal of Zoology 70:2398-2404.

259 Canter, E. J., C. Cuellar-Gempeler, A. I. Pastore, T. E. Miller, and O. U. Mason. 2018. Predator

260 identity more than predator richness structures aquatic microbial assemblages in Sarracenia

261 purpurea leaves. Ecology 99:652-660.

262 Cheng, Y.-Q., M. Yang, and A. M. Matter. 2007. Characterization of a gene cluster responsible

263 for the biosynthesis of anticancer agent FK228 in Chromobacterium violaceum No. 968. Applied

264 and Environmental Microbiology 73:3460-3469.

265 Cochran-Stafira, D. L., and C. N. von Ende. 1998. Integrating bacteria into food webs: Studies

266 with Sarracenia purpurea inquilines. Ecology 79:880-898.

267 Darby, B. J., and M. A. Herman. 2014. Effect of prey richness on a consumer's intrinsic growth

268 rate. Oecologia 175:243-250.

269 Elliott, J. M. 2003. A comparative study of the functional response of four species of carnivorous

270 stoneflies. Freshwater Biology 48:191-202.

271 Ellison, A. M., and N. J. Gotelli. 2002. Nitrogen availability alters the expression of carnivory in

272 the northern pitcher plant, Sarracenia purpurea. Proceedings of the National Academy of

273 Sciences 99:4409-4412.

274 Faria, L. D. B., W. A. C. Godoy, and L. A. Trinca. 2004. Dynamics of handling time and

275 functional response by larvae of Chrysomya albiceps (Dipt., Calliphoridae) on different prey

276 species. Journal of Applied Entomology 128:432-436. 
277 Fleischer, S. R., C. P. terHorst, and J. Li. 2018. Pick your trade-offs wisely: Predator-prey eco-

278 evo dynamics are qualitatively different under different trade-offs. Journal of Theoretical

279 Biology 456:201-212.

280 Gaines, A., M. Ludovice, J. Xu, M. Zanghi, R. J. Meinersmann, M. Berrang, W. Daley, et al.

281 2019. The dialogue between protozoa and bacteria in a microfluidic device. PLOS ONE

282 14:e0222484.

283 Goyal, A., L. S. Bittleston, G. E. Leventhal, L. Lu, and O. X. Cordero. 2021. Interactions

284 between strains govern the eco-evolutionary dynamics of microbial communities. bioRxiv

2852021.01 .04 .425224$.

286 Gray, S. M., D. M. Akob, S. J. Green, and J. E. Kostka. 2012. The bacterial composition within

287 the Sarracenia purpurea model system: Local scale differences and the relationship with the

288 other members of the food web. PLoS ONE 7:e50969.

289 Hoekman, D. 2007. Top-down and Bottom-up Regulation in a Detritus-based Aquatic Food

290 Web: A Repeated Field Experiment Using The Pitcher Plant (Sarracenia purpurea) inquiline

291 community. The American Midland Naturalist 157:52-62.

292 Holdridge, E. M., C. Cuellar-Gempeler, and C. P. terHorst. 2016. A shift from exploitation to

293 interference competition with increasing density affects population and community dynamics.

294 Ecology and Evolution 6:5333-5341.

295 Holling, C. 1959. Some characteristics of simple types of predation and parasitism. The

296 Canadian Entomologist 91:385-398.

297 Kendall, B. E., C. J. Briggs, W. W. Murdoch, P. Turchin, S. P. Ellner, E. McCauley, R. M.

298 Nisbet, et al. 1999. Why do populations cycle? A synthesis of statistical and mechanistic

299 modeling approaches. Ecology 80:1789-1805. 
Kneitel, J. M., and T. E. Miller. 2002. Resource and top-predator regulation in the pitcher plant

301 (Sarracenia purpurea) inquiline community. Ecology 83:680-688.

302 Koopman, M. M., and B. C. Carstens. 2011. The microbial phyllogeography of the carnivorous

303 plant Sarracenia alata. Microbial Ecology 61:750-758.

304 Koopman, M. M., D. M. Fuselier, S. Hird, and B. C. Carstens. 2010. The carnivorous pale

305 pitcher plant harbors diverse, distinct, and time-dependent bacterial communities. Applied and 306 Environmental Microbiology 76:1851-1860.

307 LB Solid or Liquid Medium: 2016. Cold Spring Harbor Protocols 2016:pdb.rec088203.

308 Mendiburu, F. de, and M. Yaseen. 2020. agricolae: Statistical Procedures for Agricultural

309 Research.R package.

310 Miller, T., D. Cassill, C. Johnson, C. Kindell, J. Lepis, D. Mcinnes, T. Bevis, et al. 1994.

311 Intraspecific and interspecific competition of Wyeomyia smithii (coq.) (culicidae) in pitcher plant

312 communities. American Midland Naturalist 131:136.

313 Miller, T. E., E. R. Moran, and C. P. terHorst. 2014. Rethinking niche evolution: Experiments

314 with natural communities of protozoa in pitcher plants. The American Naturalist 184:277-283.

315 Miller, T. E., and C. P. terHorst. 2012. Testing successional hypotheses of stability,

316 heterogeneity, and diversity in pitcher-plant inquiline communities. Oecologia 170:243-251.

317 Mohapatra, B. R., and K. Fukami. 2005. Effect of different bacterial species on the growth

318 kinetics of the heterotrophic nanoflagellate Jakoba libera. Basic and Applied Ecology 6:67-73.

319 Mooney, K. A., D. S. Gruner, N. A. Barber, S. A. V. Bael, S. M. Philpott, and R. Greenberg.

320 2010. Interactions among predators and the cascading effects of vertebrate insectivores on

321 arthropod communities and plants. Proceedings of the National Academy of Sciences 107:7335-

3227340. 
323 Mouquet, N., T. Daufresne, S. M. Gray, and T. E. Miller. 2008. Modelling the relationship

324 between a pitcher plant (Sarracenia purpurea) and its phytotelma community: mutualism or

325 parasitism? Functional Ecology 22:728-737.

326 Paisie, T. K., T. E. Miller, and O. U. Mason. 2014. Effects of a ciliate protozoa predator on

327 microbial communities in pitcher plant (Sarracenia purpurea) leaves. PLoS ONE 9:e113384.

328 Peckarsky, B. L., P. A. Abrams, D. I. Bolnick, L. M. Dill, J. H. Grabowski, B. Luttbeg, J. L.

329 Orrock, et al. 2008. Revisiting the classics: Considering nonconsumptive effects in textbook

330 examples of predator-prey interactions. Ecology 89:2416-2425.

331 Peterson, C. N., S. Day, B. E. Wolfe, A. M. Ellison, R. Kolter, and A. Pringle. 2008. A keystone

332 predator controls bacterial diversity in the pitcher $\square$ plant (Sarracenia purpurea) microecosystem.

333 Environmental Microbiology 10:2257-2266.

334 Pickup, Z. L., R. Pickup, and J. D. Parry. 2007. Effects of bacterial prey species and their

335 concentration on growth of the amoebae Acanthamoeba castellanii and Hartmannella

336 vermiformis. Applied and Environmental Microbiology 73:2631-2634.

337 R Core Team. 2013. R: A language and environment for statistical computing.

338 Sasidharan, A., N. K. Sasidharan, D. B. N. S. Amma, R. K. Vasu, A. V. Nataraja, and K.

339 Bhaskaran. 2015. Antifungal activity of violacein purified from a novel strain of

340 Chromobacterium sp. NIIST (MTCC 5522). Journal of Microbiology 53:694-701.

341 Singh, B. N. 1945. The selection of bacterial food by soil amoebae, and the toxic effects of

342 bacterial pigments and other products on soil protozoa. British Journal of Experimental

343 Pathology 26:316-325. 
344 Stap, I. van der, M. Vos, A. M. Verschoor, N. R. Helmsing, and W. M. Mooij. 2007. Induced

345 defenses in herbivores and plants differentially modulate a trophic cascade. Ecology 88:2474-

3462481.

347 Strom, S. L., and H. Loukos. 1998. Selective feeding by protozoa: model and experimental

348 behaviors and their consequences for population stability. Journal of Plankton Research 20:831-

349846.

350 Werner, E. E. 1974. The fish size, prey size, handling time relation in several sunfishes

351 and some implications. Journal of the Fisheries Research Board of Canada 31:1531-1536.

352 YPD media. 2010.Cold Spring Harbor Protocols 2010:pdb.rec12315-pdb.rec12315. 


\section{$357 \quad$ Figure Legends}

359 Figure 1. Protist abundance depends on the identity of bacterial strain $\left(\mathrm{F}_{(21,153)}=14.23, \mathrm{P}<\right.$

360 0.001). Box plot definitions: center line - median, upper and lower box limits - upper and lower

361 quartiles, whiskers - $1.5 \mathrm{x}$ inter-quartile range, outliers - any points outside the $1.5 \mathrm{x}$ inter-

362 quartile range.

364 Figure 2. Protist abundance varies depending on a mixture of strains $\left(F_{(4,34)}=29.46, P<0.001\right)$.

365 Any strain combination that contained harmful strains of Chromobacterium resulted in poor

366 protozoan growth. Box plot definitions: center line - median, upper and lower box limits - upper

367 and lower quartiles, whiskers - $1.5 \mathrm{x}$ inter-quartile range, outliers - any points outside the $1.5 \mathrm{x}$

368 inter-quartile range. 


\section{Figures}

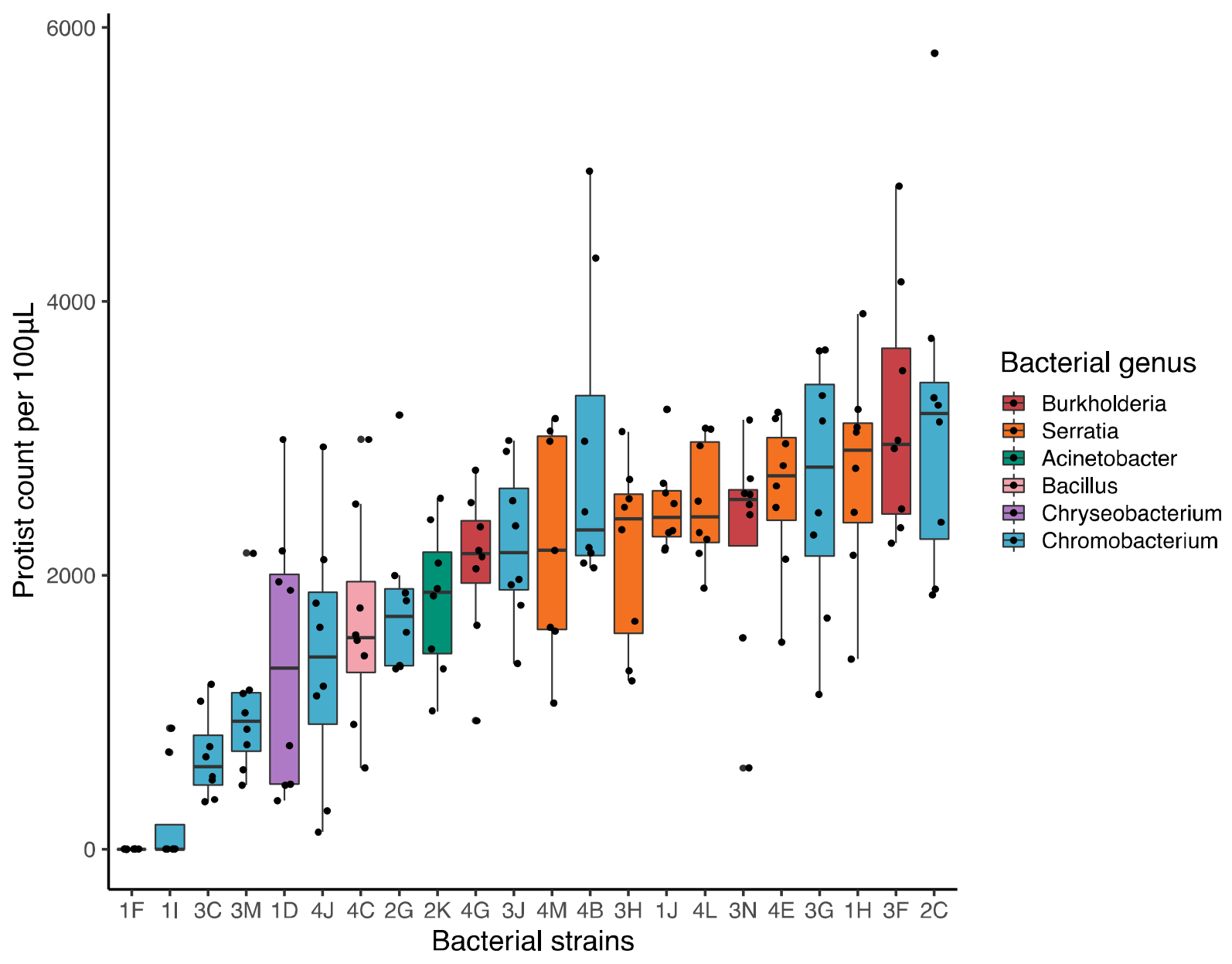

372 Figure 1. Protist abundance depends on the identity of bacterial strain $\left(\mathrm{F}_{(21,153)}=14.23, \mathrm{P}<\right.$

373 0.001). Box plot definitions: center line - median, upper and lower box limits - upper and lower

374 quartiles, whiskers - $1.5 \mathrm{x}$ inter-quartile range, outliers - any points outside the $1.5 \mathrm{x}$ inter-

375 quartile range. 


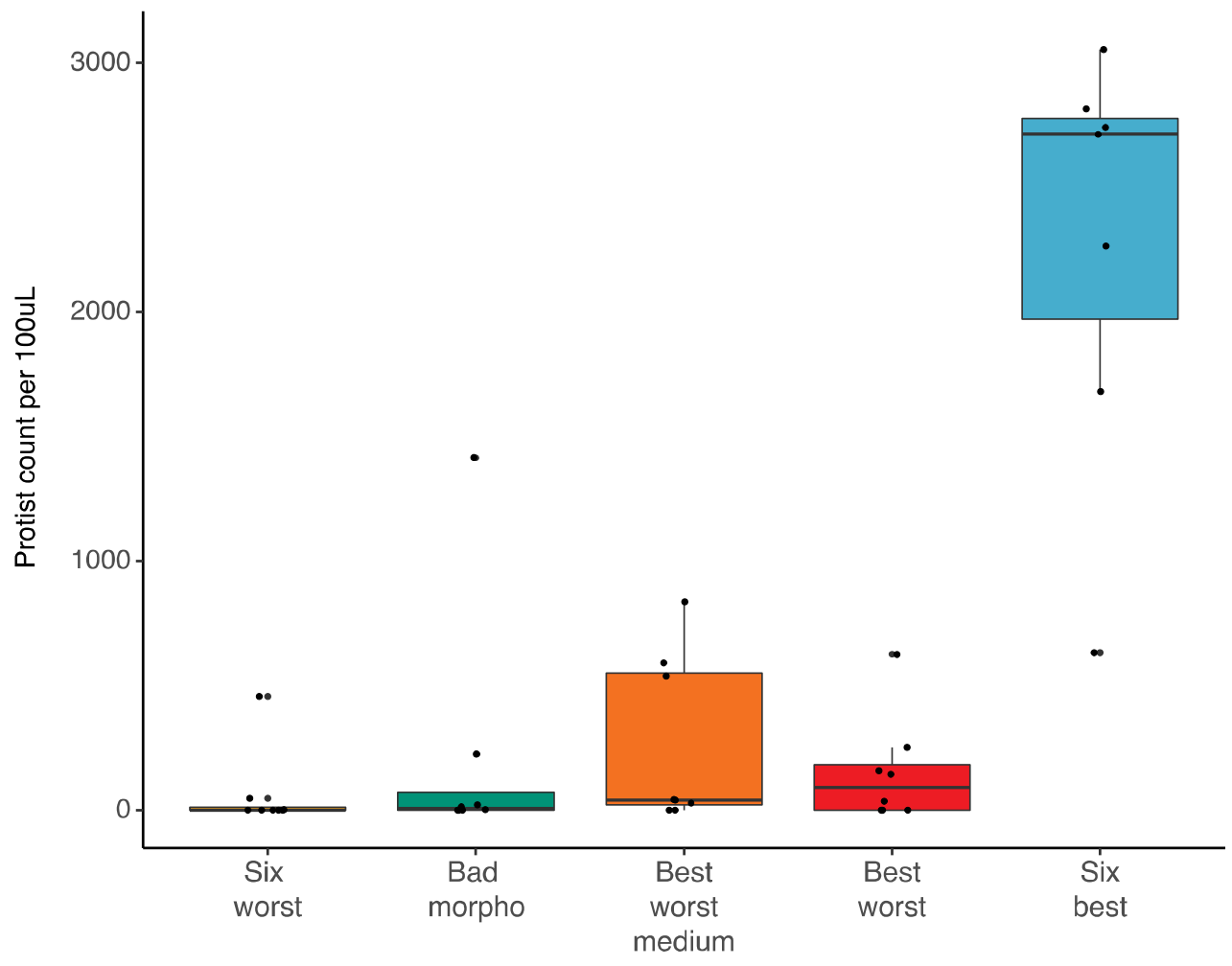

379 Figure 2. Protist abundance varies depending on a mixture of strains $\left(\mathrm{F}_{(4,34)}=29.46, \mathrm{P}<0.001\right)$.

380 Any strain combination that contained harmful strains of Chromobacterium resulted in poor

381 protozoan growth. Box plot definitions: center line - median, upper and lower box limits - upper

382 and lower quartiles, whiskers - $1.5 \mathrm{x}$ inter-quartile range, outliers - any points outside the $1.5 \mathrm{x}$

383 inter-quartile range. 